\title{
Assessing Reactive Strength Measures in Jumping and Hopping Using the Optojump ${ }^{\mathrm{TM}}$ System
}

\author{
by \\ Robin Healy ${ }^{1}$ Ian C. Kenny ${ }^{1}$, Andrew J. Harrison ${ }^{1}$
}

\begin{abstract}
The aim of this study was to assess the concurrent validity of the Optojump ${ }^{T M}$ system (Microgate, Bolzano, Italy) versus a force platform in the estimation of temporal and reactive strength measures. In two separate investigations, twenty physically active males performed double-leg and single-leg drop jumps from a box height of 0.3 $m$ and $a 10 \mathrm{~s}$ vertical bilateral hopping test. Contact time, flight time and total time (the sum of contact and flight time) were concurrently assessed during single and double-leg drop jumps and during hopping. Jump height, the reactive strength index and the reactive strength ratio were also calculated from contact time and flight time. Despite intraclass correlation coefficients (ICCs) for all variables being close to 1 (ICC >0.975), a significant overestimation was found in contact time $(0.005 \pm 0.002 \mathrm{~s})$ and underestimations in flight time $(0.005 \pm 0.003 \mathrm{~s})$, the reactive strength index $(0.04 \pm$ $0.02 \mathrm{~m} \cdot \mathrm{s}-1)$ and the reactive strength ratio $(0.07 \pm 0.04)$. Overestimations in contact time and underestimations in flight time were attributed to the physical design of the Optojump ${ }^{T M}$ system as the transmitter and receiver units were positioned $0.003 \mathrm{~m}$ above the floor level. The Optojump ${ }^{T M}$ demonstrated excellent overall temporal validity with no differences found between systems for total time. Coaches are advised to be consistent with the instrumentation used to assess athletes, however, in the case of comparison between reactive strength values collected with the Optojump ${ }^{T M}$ and values collected with a force platform, regression equations are provided.
\end{abstract}

Key words: force platform, drop jump, jump height, contact time, validity, photoelectric cells.

\section{Introduction}

Vertical jump testing is commonly used by researchers and coaches alike as a means of monitoring physical capacities of athletes and assessing the effects of training interventions (Cronin and Hansen, 2005). Although a considerable amount of research has focused on jump height $(\mathrm{JH})$ and lower body power in the squat and countermovement jumps, additional assessments such as drop jumps and hopping tests can give coaches more information about the stretch shortening cycle capacity of their athletes (Flanagan and Comyns, 2008). Dynamic movements such as jumping and sprinting require the rapid coupling of eccentric and concentric muscle contractions, i.e. the stretch shortening cycle. This form of contraction produces a much more powerful contraction than from a concentric contraction alone (Young, 1995). Reactive strength has been previously described as a measure of an individual's ability to change from an eccentric contraction to a concentric contraction (Young, 1995). It has been widely studied due to its association to sprint performance (Cronin and Hansen, 2005; Barr and Nolte, 2011), ability to monitor neuromuscular fatigue (Hamilton, 2009; Beattie and Flanagan, 2015) and ability to identify individual limb differences (Flanagan et al., 2008; Schiltz et al., 2009).

The reactivity index or reactive strength index (RSI) has commonly been used to assess an athlete's stretch-shortening cycle function and to

1 - 1. Biomechanics Research Unit, Department of Physical Education E Sport Sciences, University of Limerick, Ireland. 
evaluate athlete's rebound capabilities (Llyod et al., 2009). The RSI can be calculated by dividing $\mathrm{JH}$ by ground contact time (CT) (Flanagan and Comyns, 2008; Young, 1995) or alternatively, by dividing flight time (FT) by CT (Choukou et al., 2014; Markwick et al., 2014). For the purposes of this paper the latter method will be referred to as the reactive strength ratio (RSR) as it represents the ratio of FT achieved based on the time spent in contact with the ground. As JH is derived directly from FT, the RSR will usually yield a higher value than the RSI as the numerator, i.e. FT, will generally have a larger absolute value than $\mathrm{JH}$.

The drop jump is a plyometric exercise where an individual drops from a predetermined height and immediately on landing, performs a maximal-effort vertical jump while also trying to minimize CT (Bobbert, 1990). This can be performed with both legs or with a single leg and has been widely used to assess reactive strength (Flanagan et al., 2008; Markwick et al., 2014). Vertical bilateral hopping tests have been used extensively by researchers to assess leg and ankle stiffness (Llyod et al., 2009). Recent research found that the RSI assessed during bilateral hopping had a strong correlation to $60 \mathrm{~m}$ sprint performance, with no association found between the RSI assessed during an ankle jump and a drop jump (Nagahara et al., 2014). This suggests that coaches should consider assessing their athlete's reactive abilities through more than just one jump modality.

Although various methods exist for measuring CT and FT during jumping and hopping, the force platform has been reported as the laboratory gold standard (Bosquet et al., 2009; García-López et al., 2013; Kenny et al., 2012). Alternative devices that are relatively cheaper and mobile include: electronic jump mats and photoelectric cells (McMaster et al., 2014). Although electronic jump mats have been shown to accurately and reliably estimate FT and thus $\mathrm{JH}$, poor validity has been reported in the estimation of CT (Kenny et al., 2012; Llyod et al., 2009). Consequently, measures of reactive strength have been shown to be unreliable when electronic jump mats were used (Kenny et al., 2012; Llyod et al., 2009).

Recent research has validated the use of the Optojump ${ }^{\mathrm{TM}}$ system of photoelectric cells for estimating FT and JH during countermovement jumps and squat jumps (Castagna et al., 2013; Glatthorn et al., 2011). This system has some practical advantages over a force platform as it is less expensive, has greater mobility for field testing, provides real-time performance feedback and can be set up to operate on any flat sport specific surface, thus increasing its ecological validity (García-López et al., 2013). Although this device has been widely used to assess CT, the RSI and RSR during drop jumping (Di Cagno et al., 2013; Erčulj et al., 2009) and hopping tests (Bosquet et al., 2009; Di Cagno et al., 2009; Dupeyron et al., 2013; Girard et al., 2006), its validity to assess these measures has yet to be determined. Accordingly, there is a need to evaluate the validity of the Optojump ${ }^{\mathrm{TM}}$ system. It is hypothesized that small differences in temporal variables will compound to yield larger differences in reactive strength measures due to the mathematical dependence of the RSI and RSR on flight time and contact time.

The aim of this study therefore, was to determine the concurrent validity of the Optojump ${ }^{\mathrm{TM}}$ system of photoelectric cells, with force platform measurements of $\mathrm{CT}$, FT, the RSI and RSR in drop jumping and hopping.

\section{Material and Methods}

\section{Participants}

Twenty participants were recruited for the first investigation (mean $\pm \mathrm{SD}$, age: $23 \pm 2$ years; body height: $1.80 \pm 0.05 \mathrm{~m}$; body mass: $81 \pm$ $13 \mathrm{~kg}$ ) with a separate sample of twenty participants recruited for the second investigation (mean \pm SD, age: $22 \pm 1$ years; body height: $1.81 \pm$ $0.05 \mathrm{~m}$; body mass: $79 \pm 9 \mathrm{~kg}$ ). All participants were physically active males from a range of sports including track and field, Rugby union, soccer, hurling as well as Gaelic football, and were free of any injuries at the time of testing. The participants were familiar with double-leg and single-leg drop jumps and bilateral vertical hopping. They were also asked to refrain from any strenuous physical activity on the day before testing. Ethical approval was provided by the University of Limerick Research Ethics Committee and written consent forms were completed by all participants prior to testing. Experimental Design

In this study, two separate investigations were completed to assess the concurrent validity 
and potential interchangeability of the Optojump ${ }^{\mathrm{TM}}$ system and force platform. In the first investigation, twenty male participants were asked to perform single and double-leg drop jumps with CT, FT, JH, the RSI and RSR recorded concurrently for each jump by the Optojump ${ }^{\mathrm{TM}}$ system and force platform. In the second investigation, twenty different male participants performed a $10 \mathrm{~s}$ bilateral vertical hopping trials at a frequency of $2 \mathrm{~Hz}$ with the same measures recorded as in investigation one.

\section{Experimental Protocol}

The Optojump ${ }^{\mathrm{TM}}$ photoelectric cells (Microgate, Bolzano, Italy) consist of two parallel bars connected to a personal computer. One bar acts as a transmitter unit containing 96 light emitting diodes positioned $0.003 \mathrm{~m}$ above the ground, whereas the other acts as the receiver unit. When the light is interrupted by an individual's foot during a jump, the timer in the unit is triggered and records with a precision of 1 ms which allows the measurement of $\mathrm{CT}$ as the total time that the light is interrupted and FT as the total time between interruptions.

For the purposes of this study, the Optojump ${ }^{\mathrm{TM}}$ bars were set up one metre apart alongside dual AMTI OR6-5 force platforms operating at $1,000 \mathrm{~Hz}$, so that all jumps could be assessed by both devices concurrently (Figure 1). In both investigations, the participants performed a standardized warm up similar to previous jumping and hopping investigations (Bosquet et al., 2009; Glatthorn et al., 2011) consisting of three minutes of running at a self-selected, comfortable pace followed by two sets of ten dynamic stretches (forward and sideways hip swings, bodyweight squats, lunges) and submaximal attempts at double-leg and single-leg drop jumps or hopping.

\section{Drop Jump Tests}

In the first investigation, following a standardized warm up, participants performed double-leg drop jumps and five single-leg drop jumps on their dominant leg. The first three jumps of each jump type that were successfully recorded by both devices concurrently were selected for analysis. All jumps were performed from a box height of $0.3 \mathrm{~m}$. Strict instructions were given to each participant to keep hands on hips at all times to constrain any involvement from the upper body, avoid stepping down from the box or hopping off of the box, to avoid tucking motion in the air i.e. legs kept straight and attempt to land in the same position as take-off. The aim of the jump was to minimize CT while also attempting to achieve maximal height (Young et al., 1995). A rest period of thirty seconds was given between trials of the same jump type, with three minute rest given between different jump types to avoid any residual effects of fatigue on performance (Read and Cisar, 2001). The dependent variables calculated for both jump types were: $\mathrm{CT}, \mathrm{FT}, \mathrm{JH}$, the RSI and RSR. All variables apart from the RSR were automatically calculated and output by Optojump ${ }^{\mathrm{TM}}$ proprietary software (Optojump ${ }^{\mathrm{TM}}$ Next software, version 1.9.9.0) onto a personal computer. For the force platform, variables were calculated based on the force time trace recorded. CTs and FTs were obtained directly from the force platform data using a threshold of $>10 \mathrm{~N}$ to determine contact and $<10 \mathrm{~N}$ to determine flight. $\mathrm{JH}$ was estimated using the second mathematical equation of linear motion i.e.

$$
\begin{aligned}
& \mathrm{s}=\mathrm{ut}+1 / 2 \mathrm{at} \mathrm{t}^{2} \\
& \quad \text { where } \mathrm{s}=\mathrm{JH}, \mathrm{a}=9,81 \mathrm{~m} / \mathrm{s}^{2} \text { and } \mathrm{t}=\frac{\mathrm{FT}}{\mathrm{a}} .
\end{aligned}
$$

Hopping Test

In the second investigation, participants were permitted several trials of a $10 \mathrm{~s}$ hopping test until a valid trial was performed. A hopping frequency of $2 \mathrm{~Hz}$ ( 20 consecutive hops) was imposed through the use of a metronome operating at 120 beats per minute. Similarly to other investigations, participants were instructed to land in the same position as take-off and to keep their hands on their hips throughout (Bosquet et al., 2009). All trials were visually assessed by the same investigator to ensure consistent technique and remove invalid trials i.e. where participants did not land on the force platform or took their hands off their hips. The dependent variables calculated for hopping were identical to those calculated for drop jumps.

For all jumping $(n=120)$ and hopping trials $(n=400)$, the total time (TT) was calculated as the CT added to the FT, so that the overall temporal validity of the Optojump ${ }^{\mathrm{TM}}$ could be assessed. This allowed any potential concurrent over or underestimation of CT and FT to be determined.

\section{Statistical Analyses}

Systematic differences or bias between the Optojump ${ }^{\mathrm{TM}}$ and force platform measures were 
assessed using paired t-tests with the alpha level set at $p<0.003$ with a Bonferroni correction applied due to the number of paired t-tests carried out. Cohen's $d_{z}$ effect sizes (ES) and statistical power were calculated using $\mathrm{G}^{*}$ Power 3.1.7 (Faul et al., 2007). Effect sizes were interpreted as trivial $(\mathrm{ES}<0.2)$, small $(0.2 \leq \mathrm{ES}<$ $0.5)$, moderate $(0.5 \leq \mathrm{ES}<0.8)$ and large $(\mathrm{ES} \geq 0.8)$ according to the scale proposed by Cohen (1988). Concurrent (criterion related) validity of the Optojump ${ }^{\mathrm{TM}}$ system was examined using intraclass correlation coefficients (ICCs) $(2,1)$ with 95\% confidence intervals (CI) (Atkinson and Nevill, 1998) and Bland-Altman 95\% limits of agreement (LOA) (Bland and Altman, 1986). Based on the recommendations of Hopkins (2004), regression analysis was used to develop regression equations for both the RSI and RSR. Using the regression equations, predicted force platform values were calculated and then plotted against the residual values (differences between predicted and actual force platform values) in order to check for non-uniformity of error or heteroscedasticity. The standard error of the estimate (SEE) was calculated and expressed as a percentage of the mean force platform data to give an indication of the accuracy of the predicted values from the regression equations.

\section{Results}

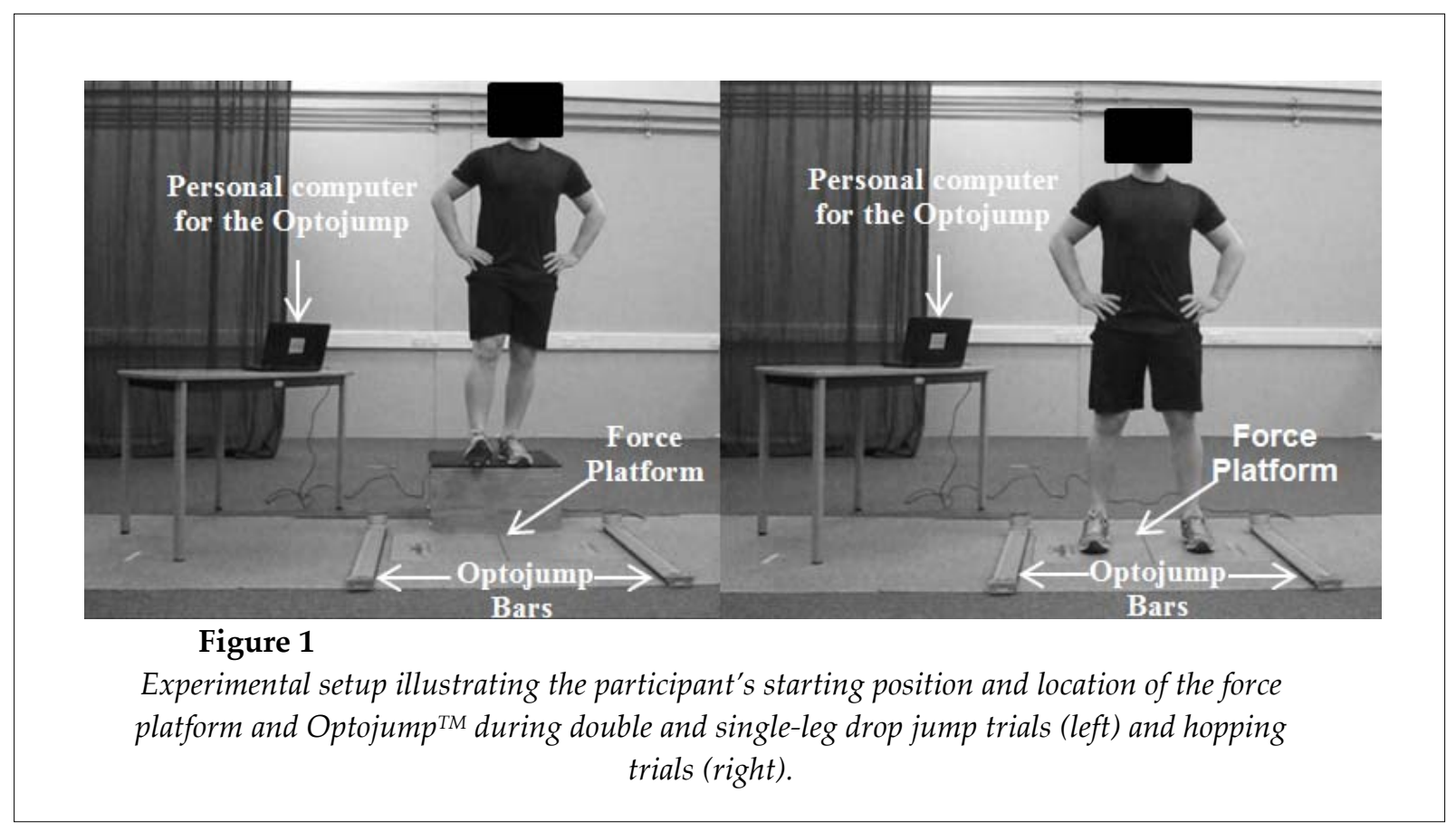

Figure 1

Experimental setup illustrating the participant's starting position and location of the force platform and Optojump ${ }^{T M}$ during double and single-leg drop jump trials (left) and hopping trials (right).
Mean results for the double and single-leg drop jumps and hopping are given in Tables 1-3, respectively. ICCs between devices for all measures were very high $(>0.975)$, however, significant differences in $\mathrm{CT}, \mathrm{FT}, \mathrm{JH}$, the RSI and RSR were found $(\mathrm{p}<0.001)$ with power $>99 \%$ and very large effect sizes (1.6 to 4.5$)$. No significant differences were found between devices for total time $(\mathrm{p}=0.828)$ with a trivial ES $(0.01)$, near perfect ICCs (0.999) and mean bias $<0.001 \mathrm{~s}$.

When all jumps and hops were combined, the mean bias $\pm 95 \%$ LOA was $0.005 \pm 0.005 \mathrm{~s}(2.44 \pm$ $2.44 \%$ ) of the mean performance for CT and -0.005 $\pm 0.005 \mathrm{~s}(-1.53 \pm 1.53 \%)$ for FT as illustrated in Figure 2. Mean bias $\pm 95 \%$ LOA was $-0.04 \pm 0.05$ $\mathrm{m} \cdot \mathrm{s}-1(-5.64 \pm 7.05 \%)$ for the RSI and $-0.07 \pm 0.08 \mathrm{~s}$ $(-4.34 \pm 4.93 \%)$ for the RSR. Differences between devices for the RSI and RSR were found to increase as the size of the measure increased. Associations between Optojump $\mathrm{P}^{\mathrm{TM}}$ and force platform RSI and RSR measures along with Bland-Altman plots are given in Figure 3. The force platform RSI and RSR were predicted by the following linear regression equations:

\section{Force platform RSI $=1.0384^{*}$ Optojump ${ }^{\mathrm{TM}} \mathrm{RSI}+0.0145$}

Force platform RSR $=1.0365^{*}$ Optojump ${ }^{\mathrm{TM}} \mathrm{RSR}+0.014$

A plot of the predicted force platform values for the RSI and RSR versus the residuals is given in Figure 4. 
Table 1

Contact time, flight time, jump height, the RSI and RSR measured during the double-leg drop jump with the Optojump ${ }^{T M}$ and the force platform. Data are presented as mean (SD).

\begin{tabular}{|c|c|c|c|c|}
\hline & Contact Time (s) & Flight Time & $\begin{array}{c}\text { RSI } \\
\left(\mathrm{m} \cdot \mathrm{s}^{-1}\right)\end{array}$ & RSR \\
\hline Optojump ${ }^{\mathrm{TM}}$ & $0.218(0.030)$ & $0.470(0.046)$ & $1.28(0.30)$ & $2.20(0.38)$ \\
\hline Force Platform & $0.214(0.030)^{*}$ & $0.474(0.046)^{*}$ & $1.33(0.31)^{*}$ & $2.27(0.39)^{*}$ \\
\hline Bias $\pm 95 \%$ LOA & $0.004 \pm 0.002$ & $-0.004 \pm 0.002$ & $-0.05 \pm 0.04$ & $-0.07 \pm 0.05$ \\
\hline $\begin{array}{l}\text { Effect Size Cohen's } \\
\qquad \mathrm{d}_{\mathrm{z}}\end{array}$ & 3.0 & 1.9 & 3.0 & 2.8 \\
\hline ICC & 0.989 & 0.995 & 0.985 & 0.983 \\
\hline$(95 \% \mathrm{CI})$ & $(0.982-0.993)$ & $(0.992-0.997)$ & $(0.973-0.997)$ & $(0.970-0.996)$ \\
\hline
\end{tabular}

*Significantly different from the Optojump ${ }^{T M}(p<0.001)$ LOA: 95\% Limits of agreement

Table 2

Contact time, flight time, jump height, the RSI and RSR measured during the single-leg drop jump with the Optojump ${ }^{T M}$ and the force platform. Data are presented as mean (SD).

\begin{tabular}{|c|c|c|c|c|}
\hline & Contact Time $\quad$ (s) & Flight Time & $\begin{array}{c}\text { RSI } \\
\left(\mathrm{m} \cdot \mathrm{s}^{-1}\right)\end{array}$ & RSR \\
\hline Optojump ${ }^{\mathrm{TM}}$ & $0.292(0.033)$ & $0.329(0.052)$ & $0.47(0.15)$ & $1.19(0.23)$ \\
\hline Force Platform & $0.285(0.032)^{*}$ & $0.336(0.050)^{*}$ & $0.50(0.15)^{*}$ & $1.15(0.23)^{*}$ \\
\hline Bias $\pm 95 \%$ LOA & $0.006 \pm 0.006$ & $-0.007 \pm 0.006$ & $-0.03 \pm 0.02$ & $-0.05 \pm 0.04$ \\
\hline $\begin{array}{l}\text { Effect Size Cohen's } \\
\qquad \mathrm{d}_{\mathrm{z}}\end{array}$ & 2.3 & 1.8 & 4.5 & 2.4 \\
\hline $\mathrm{ICC}$ & 0.978 & 0.989 & 0.982 & 0.976 \\
\hline$(95 \% \mathrm{CI})$ & $(0.964-0.987)$ & $(0.982-0.994)$ & $(0.969-0.989)$ & $(0.964-0.988)$ \\
\hline
\end{tabular}

*Significantly different from the OptojumpTM $(p<0.001)$ LOA: 95\% Limits of agreement

Table 3

Contact time, flight time, jump height, the RSI and RSR measured during continuous hopping with the Optojump ${ }^{T M}$ and the force platform. Data are presented as mean (SD).

\begin{tabular}{|c|c|c|c|c|}
\hline & Contact Time (s) & Flight Time & $\begin{array}{l}\text { RSI } \\
\left(\mathrm{m} \cdot \mathrm{s}^{-1}\right)\end{array}$ & RSR \\
\hline Optojump ${ }^{\mathrm{TM}}$ & $0.198(0.034)$ & $0.299(0.041)$ & $0.61(0.28)$ & $1.59(0.50)$ \\
\hline Force Platform & $0.192(0.033)^{*}$ & $0.304(0.040)^{*}$ & $0.65(0.29)^{*}$ & $1.67(0.52)^{*}$ \\
\hline Bias $\pm 95 \%$ LOA & $0.006 \pm 0.005$ & $-0.006 \pm 0.005$ & $-0.04 \pm 0.05$ & $-0.08 \pm 0.09$ \\
\hline $\begin{array}{l}\text { Effect Size Cohen's } \\
\qquad \mathrm{d}_{\mathrm{z}}\end{array}$ & 2.2 & 1.8 & 1.7 & 1.6 \\
\hline ICC & 0.983 & 0.989 & 0.986 & 0.985 \\
\hline$(95 \% \mathrm{CI})$ & $(0.980-0.986)$ & $(0.987-0.991)$ & $(0.981-0.991)$ & $(0.975-0.995)$ \\
\hline
\end{tabular}

${ }^{*}$ Significantly different from the Optojump ${ }^{T M}(p<0.001)$ LOA: $95 \%$ Limits of agreement 

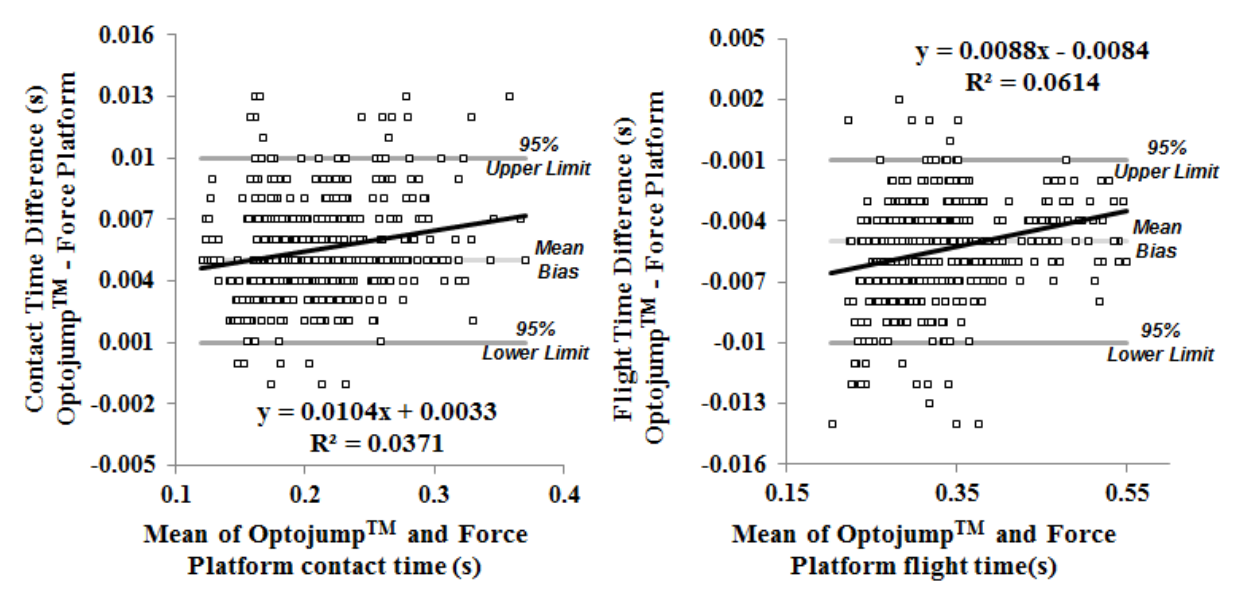

Figure 2

Bland and Altman plots of contact time measured by Optojump ${ }^{T M}$ method against Force Platform $(n=520)$ (left) and flight time Optojump ${ }^{\text {TM }}$ method against Force Platform $(n=520)$ (right).
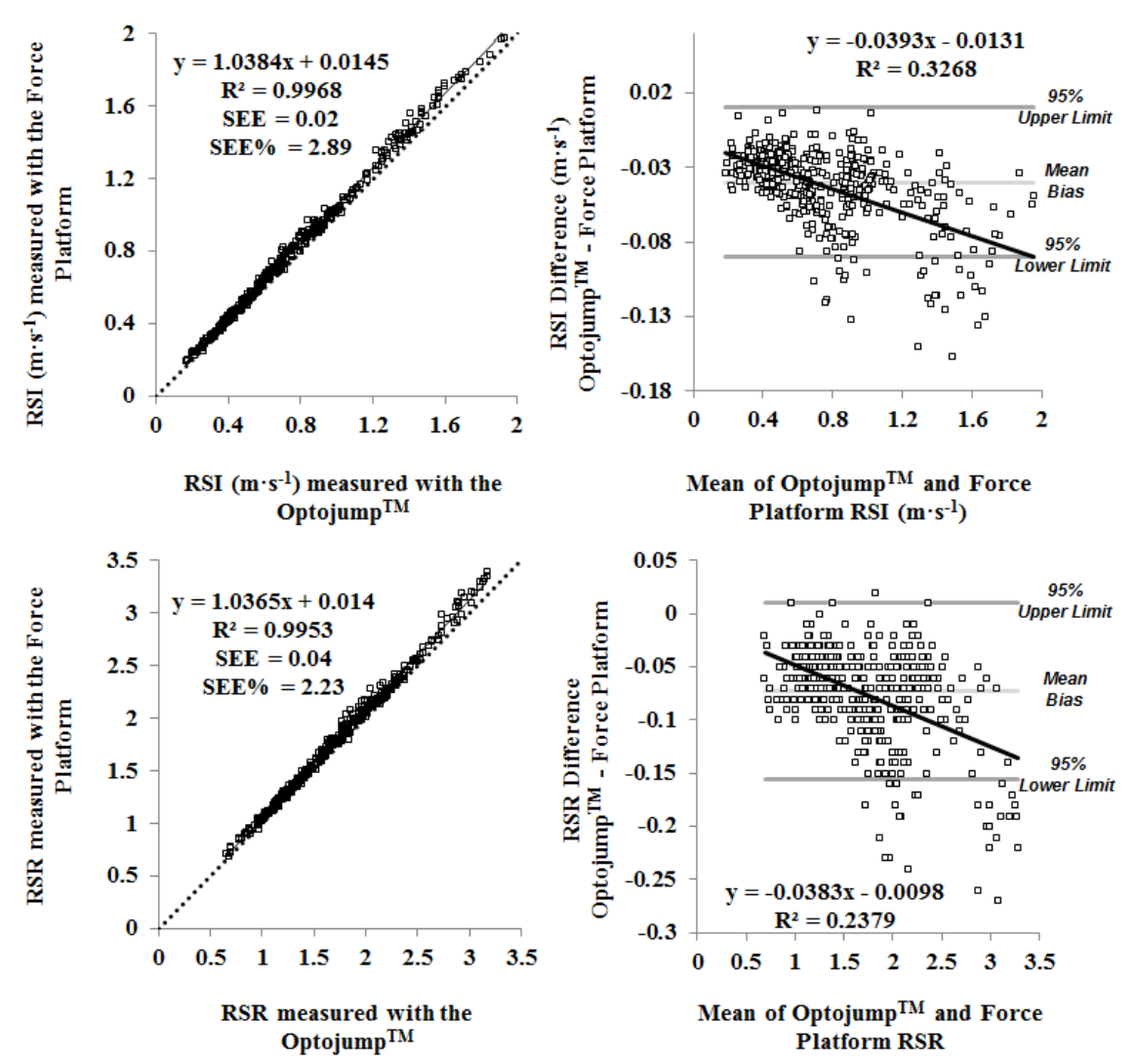

Figure 3

Left Panel: Association between Optojump ${ }^{T M}$ and force platform measures for the RSI and RSR. The dotted line is the line of identity and the black line is the trend line. SEE = Standard error of the estimate. SEE \% = Standard error of the estimate as a percentage of mean force platform values. Right panel: Bland-Altman plots for the comparison between Optojump ${ }^{T M}$ and force platform measures of the RSI and RSR. The black line is the trend line, the light grey line is the mean bias and dark grey lines are the 95\% upper and lower limits of agreement. 


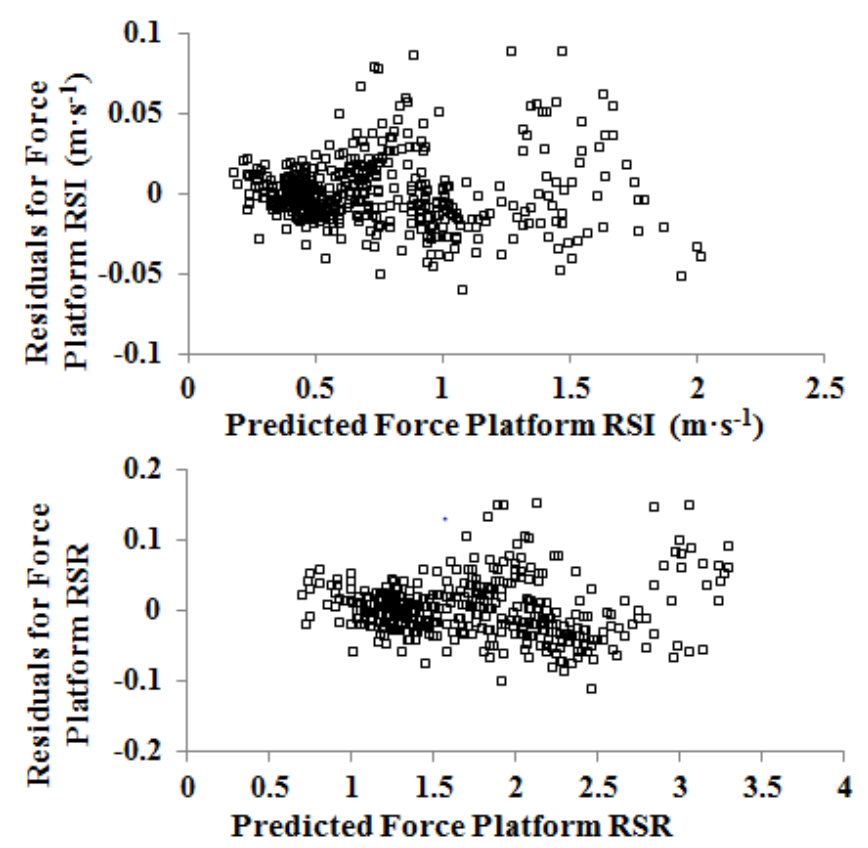

Figure 4

Scatter plot of predicted force platform values for the RSI (top) and RSR (bottom) against residuals.

\section{Discussion}

This is the first study to assess the validity of the Optojump ${ }^{\mathrm{TM}}$ system in the calculation of $\mathrm{CT}$, TT, the RSI and RSR. The results illustrate that the Optojump ${ }^{\mathrm{TM}}$ had excellent overall temporal validity, but consistently underestimated double and single-leg drop jump and hopping measures of the RSI and RSR by $3.8-6.2 \%$ and $3.1-4.8 \%$. This underestimation can be explained by differences in the calculation of CT and FT; i.e. the underlying measures that are used to calculate both the RSI and RSR. CT was found to be overestimated, whereas FT was underestimated. These differences can be attributed to the physical design of the Optojump ${ }^{\mathrm{TM}}$ system as the transmitter and receiver units were positioned $0.003 \mathrm{~m}$ above the floor level. As the force platform was embedded into the floor, this created a difference between the surface of the force platform and the photoelectric cells of the Optojump ${ }^{\mathrm{TM}}$. This discrepancy resulted in the early detection of CT and delayed detection of FT relative to the force platform. This delayed detection of FT relative to the force platform had been reported in previous research (Castagna et al., 2013). No difference was found for total time (the sum of CT and FT) which suggests that any overestimation in CT was accounted for by a subsequent underestimation in FT.

The calculation of the RSI and RSR requires $\mathrm{FT}$ and $\mathrm{CT}$. As $\mathrm{JH}$ is derived directly from FT, an underestimation in FT would result in a subsequent underestimation of $\mathrm{JH}$. In an investigation similar to the present study, Castagna et al. (2013) reported a $0.006 \mathrm{~s}$ mean difference between a force platform and Optojump ${ }^{\mathrm{TM}}$ in the calculation of FT during other forms of vertical jumping, i.e. countermovement jumps and squat jumps. This investigation found a higher CT (1.87-3.13\%) combined with a lower FT (0.84-2.08\%). These errors are compounded when the RSI and RSR are calculated leading to larger differences in the RSI 
(3.8-6.2\%) and RSR (3.1-4.8\%) compared to the force platform.

Researchers and coaches should be aware that when using the Optojump ${ }^{\mathrm{TM}}$, there may be a mean error $\pm \mathrm{SD}$ of $5.6 \pm 2.8 \%$ and $4.3 \pm 2.5 \%$ in the estimation of the RSI and RSR, respectively, compared to force platform measures. The increasing deviation between the trend line and the line of identity shown in Figure 3 and the steepness of the slopes in the Bland-Altman plots for the RSI and RSR illustrate that the error between devices generally increases as the magnitude of the measure increases i.e. proportional bias. If coaches wish to compare values measured with the Optojump ${ }^{\mathrm{TM}}$ to values collected with a force platform, the regression equations given earlier should be applied. By applying the regression equations, the standard error was reduced to $\pm 2.9 \%$ for the RSI and $\pm 2.2 \%$ for the RSR. Inspection of the scatterplots in Figure 4 illustrates that for both measures, the proportional bias can be removed, i.e. the error no longer increases with increasing values.

\section{Conclusion}

Coaches are increasingly using jumping and hopping tests to evaluate their athletes. Although the traditional focus of coaches has been on the $\mathrm{JH}$ during a squat jump or a countermovement jump, more sophisticated tests are required in order to assess reactive strength. This study found that the Optojump ${ }^{\mathrm{TM}}$ system demonstrated excellent overall temporal validity, however, CT was consistently overestimated and FT was consistently underestimated. These differences do not represent measurement error however and are simply due to the physical design of the Optojump ${ }^{\mathrm{TM}}$ system with overestimations in CT resulting in subsequent underestimations in FT. The Optojump ${ }^{\mathrm{TM}}$ is therefore a valid system to assess reactive strength abilities in athletes. Coaches are advised to be consistent with the type of a measurement system they use to assess their athletes. Coaches wishing to compare reactive qualities measured with a force platform are advised to correct the values according to the following equations: force platform RSI $=1.0384^{*}$ Optojump ${ }^{\mathrm{TM}} \mathrm{RSI}+0.0145$ and force platform RSR $=1.0365^{\circ}$ Optojump ${ }^{\text {TM }}$ RSR +0.014 .

\section{Acknowledgements}

The authors have no conflict of interest to disclose and would like to thank the Irish Research Council for financially supporting this research.

\section{References}

Atkinson G, Nevill AM. Statistical methods for assessing measurement error (reliability) in variables relevant to sports medicine. Sports Med, 1998; 26: 217-238

Barr MJ, Nolte VW. Which measure of drop jump performance best predicts sprinting speed? J Strength Cond Res, 2011; 25: 1976-1982

Beattie K, Flanagan EP. Establishing the reliability \& meaningful change of the drop-jump reactive strength index. J Aust Strength Cond Res, 2015; 23: 12-18

Bland JM, Altman D. Statistical methods for assessing agreement between two methods of clinical measurement. Lancet,1986; 1: 307-310

Bobbert MF. Drop jumping as a training method for jumping ability. Sports Med, 1990; 9: 7-22

Bosquet L, Berryman N, Dupuy O. A comparison of 2 optical timing systems designed to measure flight time and contact time during jumping and hopping. J Strength Cond Res, 2009; 23: 2660-2665

Castagna C, Ganzetti M, Ditroilo M, Giovannelli M, Rocchetti A, Manzi V. Concurrent validity of vertical 
jump performance assessment systems. J Strength Cond Res, 2013; 27: 761-768

Choukou M-A, Laffaye G, Taiar R. Reliability and validity of an accelerometric system for assessing vertical jumping performance. Biology of Sport, 2014; $31: 55$

Cohen J. Statistical Power Analysis for the Behavioral Sciences. Hillsdale, NJ: Lawrence Erlbaum Associates; 1988

Cronin JB, Hansen KT. Strength and power predictors of sports speed. J Strength Cond Res, 2005; 19: 349-357

Di Cagno A, Baldari C, Battaglia C, Monteiro MD, Pappalardo A, Piazza M, Guidetti L. Factors influencing performance of competitive and amateur rhythmic gymnastics-Gender differences. J Sci Med Sport, 2009; 12: 411-416

di Cagno A, Battaglia C, Giombini A, Piazza M, Fiorilli G, Calcagno G, Pigozzi F, Borrione P. Time of dayeffects on motor coordination and reactive strength in elite athletes and untrained adolescents. J Sport Sci Med, 2013; 12: 182

Dupeyron A, Hertzog M, Micallef J-P, Perrey S. Does an abdominal strengthening program influence leg stiffness during hopping tasks? J Strength Cond Res, 2013; 27: 2129-2133

Erčulj F, Blas M, Čoh M, Bračič M. Differences in motor abilities of various types of European young elite female basketball players. Kinesiology, 2009; 41: 203-211

Faul F, Erdfelder E, Lang A-G, Buchner A. G* Power 3: A flexible statistical power analysis program for the social, behavioral, and biomedical sciences. Behav Res Methods, 2007; 39: 175-191

Flanagan EP, Comyns TM. The use of contact time and the reactive strength index to optimize fast stretchshortening cycle training. Strength Cond J, 2008; 30: 32-38

Flanagan EP, Galvin L, Harrison AJ. Force production and reactive strength capabilities after anterior cruciate ligament reconstruction. J Athl Training, 2008; 43: 249

García-López J, Morante JC, Ogueta-Alday A, Rodríguez-Marroyo JA. The type of mat (contact vs. photocell) affects vertical jump height estimated from flight time. J Strength Cond Res, 2013; 27: 1162-1167

Girard O, Lattier G, Micallef J-P, Millet GP. Changes in exercise characteristics, maximal voluntary contraction, and explosive strength during prolonged tennis playing. Brit J Sport Med, 2006; 40: 521-526

Glatthorn JF, Gouge S, Nussbaumer S, Stauffacher S, Impellizzeri FM, Maffiuletti NA. Validity and reliability of Optojump photoelectric cells for estimating vertical jump height. J Strength Cond Res, 2011; 25: 556560

Hamilton D. Drop jumps as an indicator of neuromuscular fatigue and recovery in elite youth soccer athletes. J Aust Strength Cond Res, 2009; 17: 3-8

Hopkins WG. Bias in Bland-Altman but not regression validity analyses. Sportscience, 2004; 8: $42-46$

Kenny IC, Ó Cairealláin A, Comyns TM. Validation of an electronic jump mat to assess stretch-shortening cycle function. J Strength Cond Res, 2012; 26: 1601-1608

Lloyd RS, Oliver JL, Hughes MG, Williams CA. Reliability and validity of field-based measures of leg stiffness and reactive strength index in youths. J Sport Sci, 2009; 27: 1565-1573

Markwick WJ, Bird SP, Tufano JJ, Seitz LB, Haff GG. The Intraday Reliability of the Reactive Strength Index Calculated From a Drop Jump in Professional Men's Basketball. Int J Sports Physiol Perform, 2014; 10: $482-488$

McMaster DT, Gill N, Cronin J, McGuigan M. A brief review of strength and ballistic assessment methodologies in sport. Sports Med, 2014; 44: 603-623

Nagahara R, Naito H, Miyashiro K, Morin J, Zushi K. Traditional and ankle-specific vertical jumps as strength-power indicators for maximal sprint acceleration. J Sports Med Phys Fitness, 2014; 54: 691-699

(C) Editorial Committee of Journal of Human Kinetics 
Read MM, Cisar C. The influence of varied rest interval lengths on depth jump performance. J Strength Cond Res, 2001; 15: 279-283

Schiltz M, Lehance C, Maquet D, Bury T, Crielaard J-M, Croisier J-L. Explosive strength imbalances in professional basketball players. J Athl Training, 2009 44: 39

Young W. Laboratory strength assessment of athletes. New Stud Athlete, 1995; 10: 89-89

Young WB, Pryor JF, Wilson GJ. Effect of Instructions on characteristics of Countermovement and Drop Jump Performance. J Strength Cond, 1995; Res 9: 232-236

\section{Corresponding author:}

\section{Robin Healy}

Biomechanics Research Unit

Department of Physical Education \& Sport Sciences

University of Limerick

Ireland

Phone: +353 61234715

E-mail: robin.healy@ul.ie 